\title{
Pneumatically-driven stretching machine for ankle dorsiflexion: safety concepts and effectiveness test involving healthy young subjects
}

\author{
Yuma Shiraishi ${ }^{1 \dagger}$, Shogo Okamoto ${ }^{{ }^{*}+} \mathbb{0}$, Naomi Yamada ${ }^{1,2 \dagger}$, Koki Inoue ${ }^{1 \dagger}$, Yasuhiro Akiyama ${ }^{1}$ and Yoji Yamada
}

\begin{abstract}
Stretching is a major treatment for restoring the ankle range of motion while decreasing the ankle stiffness. An automatic stretching machine can provide long-term continual stretching at home. However, no commercial stretching machines are currently available, and therefore, no safety guidelines exist for them. In this study, we prototyped a stretching machine using a chassis from a commercial foot exerciser for preventing disuse syndrome, and then proposed three safety concepts as general requirements for stretching machines. The concepts were determined based on risk assessments, which included (1) preventing excessive force from being applied to the foot, (2) responding to the ankle stiffness and the range of motion of each different individual, and (3) preventing unintended operations by the user. The above concepts allowed us to develop a stretching machine taking into account safety measures. Further, the stretching effect of the prototype was tested on sixteen healthy young subjects ( $22.3 \pm 3.6$ years). We found that the passive resistance of the subjects at specific dorsiflexion angles decreased significantly after stretching the ankle using the prototyped machine.
\end{abstract}

Keywords: Ankle contractures, Stretching machine, Safety concepts

\section{Introduction}

Ankle contracture is a common after effect of diseases and injuries such as ankle fracture, stroke, or cerebral palsy. It is caused by the stiffened soft tissues comprising muscles and tendons around the joint, which reduces the ankle range of motion (ROM). Stretching is performed as physical therapy to improve the ROM and decrease the ankle stiffness. Long-term stretching is essential for healing ankle contracture because it may continue to develop even after discharge from the hospital, especially for stroke patients [1]. However, it is difficult for patients to receive continual stretching by physical therapists after

\footnotetext{
*Correspondence: okamoto-shogo@mech.nagoya-u.ac.jp

†Yuma Shiraishi and Shogo Okamoto contributed equally to this work ${ }^{\dagger}$ Naomi Yamada and Koki Inoue contributed equally to this work

${ }^{1}$ Graduate School of Engineering, Nagoya University, Nagoya, Japan Full list of author information is available at the end of the article
}

discharge from a hospital. Also, there are restrictions on rehabilitation opportunities because of medical insurance systems. Several devices for non-therapeutic purposes are currently available for in-home stretching. For example, a tilt board is used for stretching ankle plantar flexors. Though users are required to just stand on the tilt board, it is difficult for some elderly people to use because of balance-stability requirements. Automated stretching machines can help to solve such difficulties.

There have been few reports on ankle stretching machines. Zhang et al., Zhou et al. and Toda et al. developed machines for dorsiflexing the foot for stretching using an electro-magnetic motor or linear actuator [2-7]. Yamada et al. developed a machine for three-dimensionally controlling the foot using wire-driven mechanisms to stretch the muscles related to equinovarus $[8,9]$. This machine moves the foot by pulling two wires with 
McKibben type pneumatic actuators, which are independently attached to the inside and outside of the foot plate.

Other ankle rehabilitation machines can be classified into two groups; relying on whether they utilize voluntary ankle movement or not.

First, the machines that do not involve user's voluntary motions typically move the foot within the ankle ROM to prevent ankle contractures, disuse syndrome or thrombus. Saga et al. [10] and Cordo et al. [11] developed machines for ankle dorsiflexion or/and plantar flexion using a pneumatic actuator or an electro-magnetic motor. Others aimed to increase the blood flow of the lower limb [12-14]. For example, Sasanuma et al. [13] developed a sock-type ankle exercise machine driven by pneumatics that moves the ankle with the tip of the foot in the dorsi/plantar flexion direction. Note that these abovementioned studies were not designed to treat contractures.

Second, machines involving voluntary movement apply resistive or assistive force during the voluntary foot movement to improve muscle strength or/and motor function. Burdea et al. developed a Stewart-platform type ankle exercise machine driven by double acting type pneumatic actuators [15-18]. The patient fixes the foot to this machine and voluntarily moves the foot in the dorsi/plantar flexion direction as well as the in/ eversion direction. Another ankle rehabilitation robot covered various exercises including voluntary movement training [19]. Moreover, training machines were designed to strengthen the tibialis anterior [20] or to encourage patients' active participation [21].

Only a few studies clearly mentioned safety measures for preventing pain in the foot or the ankle. Toda et al. [6] and Sasanuma et al. [13] implemented fuse-like mechanisms for preventing excessive force from being applied to the foot. For these machines, the force transmission mechanisms encompass mechanical elements that break at certain force thresholds. In another study, passive degree of freedom was introduced to prevent misalignment of the ankle rotation axis and the machine axis [12]. However, these measures were not against hazards identified in risk assessment.

Despite the above studies, as far as we have studied, commercial stretching machines designed following safety guidelines are yet to be developed and no safety standards for ankle stretching machines exist. Additionally, no previous studies on ankle stretching machines have proposed any safety concepts or measures for such machines. However, the key for developing practical stretching machines is to reduce the risks of foot pain owing to the application of an excessive load to the foot, as well as being able to use the machine safely. In general, the safety is defined as a state with no unacceptable risks, which is realized by repetitive risk assessment and risk reduction procedures [22].

In this study, we propose safety concepts tailored to ankle stretching machines based on the risk assessment of a commercial foot exercise machine. Then, we redesign the machine as a stretching machine for ankle dorsiflexion following proposed safety concepts. Further, this paper reports the safety design of the prototype machine, and the effectiveness of the prototype stretching machine is experimentally tested on sixteen healthy young subjects. The experimental verification is essential to confirm that the designed prototype achieves the stretching effect. The experiments were conducted with the approval of the Institutional Review Board of the School of Engineering, Nagoya University (\#18-2).

\section{Redesigning of the stretching machine Overview of commercially available foot exerciser}

The stretching machine prototype is based on a commercial foot exerciser for the plantar and dorsal flexion of the ankle (Relegs, LAP Co., Ltd., Japan). Relegs machine does not have a stretching function and is used to prevent disuse syndrome, by repetitively moving a foot that cannot be voluntarily because of a stroke or injury.

Relegs is driven by a pneumatic actuator (bellows). As shown in Fig. 1, the bellows inflates in the longitudinal direction when pressurized. This deformation of the bellows rotates the machine's moving part around the rotation axis near the ankle, which, in turn, dorsiflexes the foot. Similarly, the bellows contracts when the internal pressure of the bellows decreases, which moves the foot in the plantar flexion direction.

Some foot joint exercise systems are driven by an electro-magnetic motor that enables highly responsive, precise control of a machine. However, an electro-magnetic motor brings the risk that the foot can get stuck in the emergency stop mode owing to the friction generated in the reduction gear. Of course, this drawback can be overcome using various mechanisms. In the case of a pneumatic actuator, load on the foot is eliminated by the

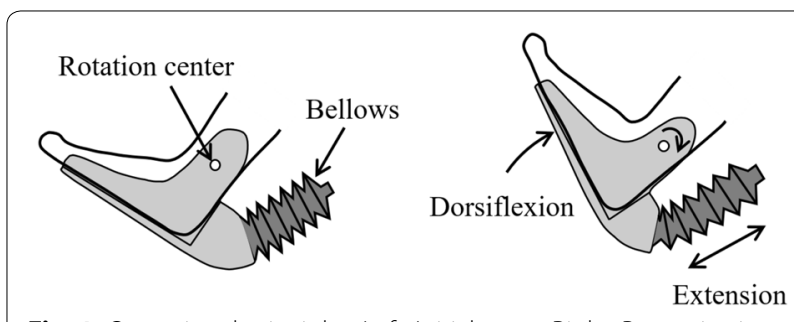

Fig. 1 Operational principles. Left: Initial stage. Right: Pressurization causes bellows to extend longitudinally, leading the machine's moving part to rotate around the rotation center (Adapted from [23]) 
decrease in the internal pressure after the air in the bellows has been exhausted. In other words, the machine is inherently safe, as the abovementioned risk can be readily reduced by designing the system such that it automatically exhausts the air during emergency stops.

\section{Prototype stretching machine}

While Relegs machine can move the foot within the ankle ROM, it does not have a stretching function. Therefore, the Relegs system was redesigned as follows.

Primarily, a stretching function was implemented. Following manual stretching by a physical therapist, the foot is dorsiflexed till the end of the ankle ROM. Subsequently, the therapist applies an additional load on the foot and maintains the foot in this posture [24]. To simulate this process, the machine was controlled such that it dorsiflexed the foot slowly and subsequently maintained this dorsiflexed posture. In the case where the ankle ROM of the user is restricted owing to diseases or other reasons, a mechanical limiter is used to mechanically restrict the angle of dorsiflexion. The aluminum legs were installed at the front bottom of the machine, as shown in Fig. 2, to change the entire machine's angle and keep the user's knee extended.

Stretching requires a pushing force in the dorsiflexion direction to extend the plantar flexor muscles effectively. The output of the Relegs system is not enough for this purpose. Thus, an air compressor (CP-12Si, Minato Electrical Co., Ltd., Japan), which can output a pressure greater than $100 \mathrm{kPa}$, was used for the driving system. The pneumatic force applied to the bellows was continuously controlled using an electro-pneumatic regulator (ITV1030-312S, SMC Co., Ltd., Japan), which itself was controlled by a microcomputer (mbed LPC1768, ARM Holdings PLC., UK).

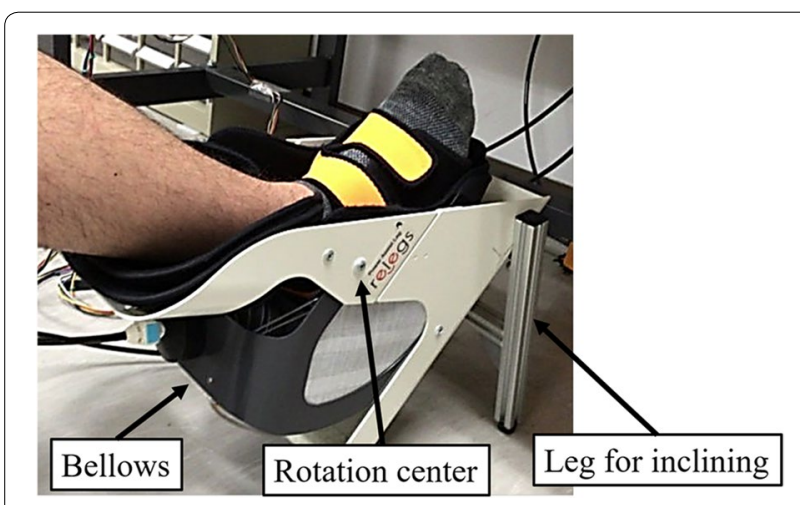

Fig. 2 Prototype of stretching machine for ankle dorsiflexion. The foot is dorsiflexed slowly and maintained in dorsiflexed posture (Adapted from [23])
The user operates the stretching machine using a three-position enabling switch (A4EG-BM2B041, OMRON Co., Ltd., Japan), which is in line with the concept of enabling devices in the field of machine safety $[25,26]$. The machine is operated continuously only when the user clearly indicates an intent to use the machine. The risk of foot pain during the machine's operation is reduced through manual operation using the enabling switch. Thus, patients who would obviously find it difficult to control our machine and cannot use it are those with paralyzed upper body parts or sensory impairments.

\section{Safety concepts and safety measures for ankle stretching machines \\ Risk assessments}

An expert on machine safety, a physical therapist, and the developers of the prototype conducted risk assessments over our prototype machine and took safety measures to reduce the unacceptable and conditionally acceptable risks, following the procedures in [22]. This process was repeated thrice until there were no more unacceptable risks. Some of the 46 risks are listed in Table 1. The listed risks were once judged as unacceptable or conditionally acceptable and are described as follows: (a) An excessive torque is applied to the ankle, causing foot pain when the machine is working. (b) The foot is moved more than the natural ROM of the ankle when the machine is working. (c) The user tries to reach a controller placed away from the body and falls, resulting in pain in the fixed ankle. (d) The foot is fixed in a non-relaxed posture owing to machine malfunction. (e) The stop button does not work correctly. (f) The user's fingers get pinched between the gaps of the machine when the machine is working. $(\mathrm{g})$ The entire machine slides on the floor and moves during use. In this case, the relative position of the user and the stretching machine changes, and an excessive load is applied to the foot or leg.

The risks judged as acceptable during the risk assessment process include abrasion of the skin on the foot near the belt used for fixing the foot. According to ISO 13482 [27], the risk of scratches on the body area where the user comes in contact with the machine must be taken into account. Mao et al. studied methods for verifying such risks for a physical assistant robot [28, 29]. Further, studies on stretching have reported that many adverse events, such as numbness and blistering, could possibly occur [30]. Cordo et al. [11] developed a foot joint exercise apparatus and tested it on 20 subjects, who performed $30 \mathrm{~min}$ of day training using the machine at home for 6 months (i.e., for more than 2000 h). During this period, only one event of skin abrasion was reported. 


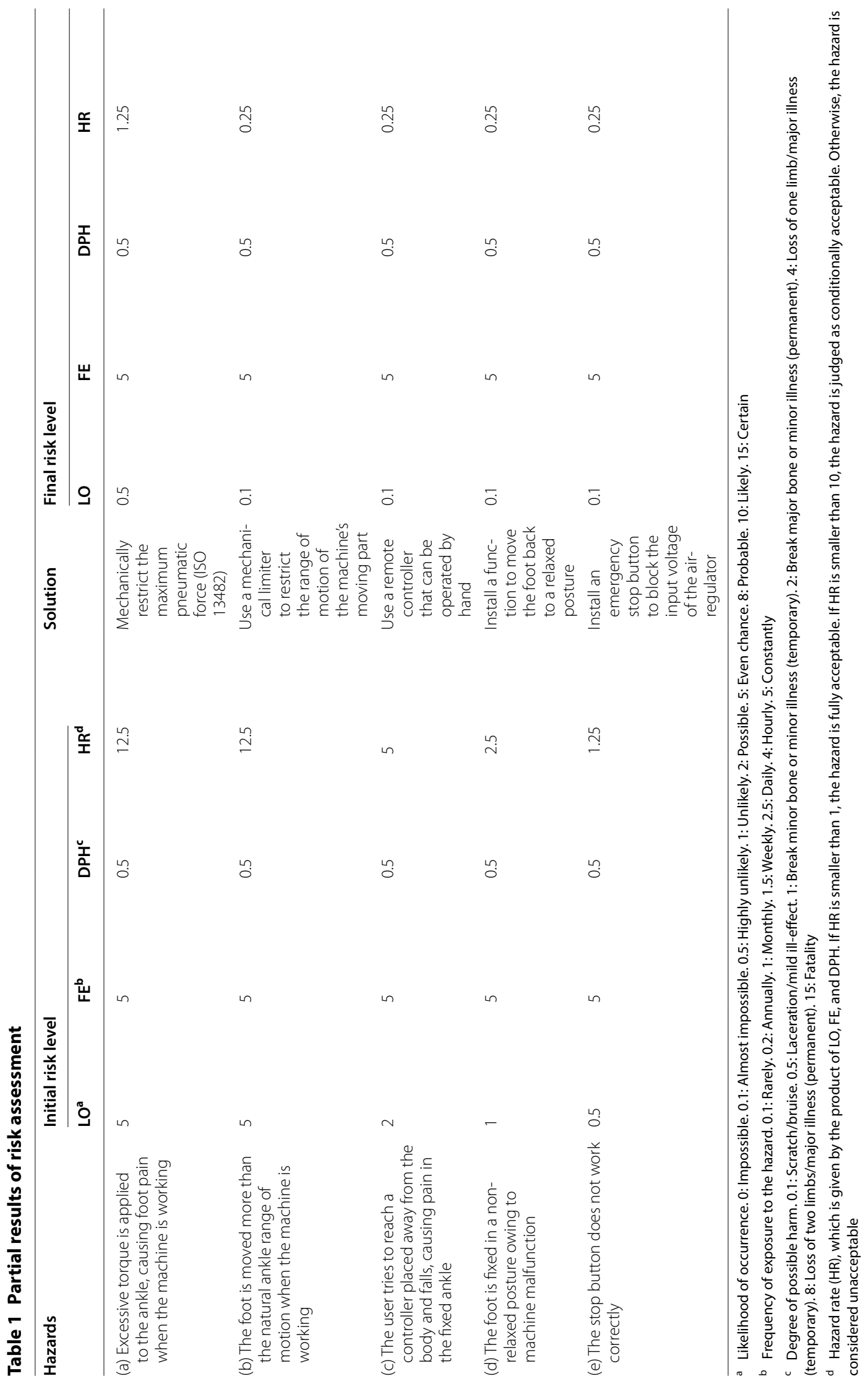


In our study, as mentioned later, no participants experience skin abrasion after using the machine for more than $50 \mathrm{~h}$.

\section{Safety concepts for stretching machines}

Most of the abovementioned unacceptable risks should be handled in accordance with existing safety standards. For example, gaps in the mechanically moving parts should be treated by following ISO 13854 [31]. Here, we summarize the risks and countermeasures related to ankle stretching machines. We propose the following three safety concepts based on the risks identified during the risk assessment process. (1) Prevent excessive loads from being applied to the ankle. (2) Deal with the individual differences in the ankle ROM and stiffness of the users. (3) Prevent involuntary operations of the machine. By implementing suitable safety measures based on these concepts, the risk of foot pain is reduced. Because there are no existing safety standards for stretching machines, the risk reduction features for these machines must be based on referable safety measures.

Regarding Concept 1 , as per the standard for personal care robots, ISO 13482 [27], the safety is stated regarding the force control when a human and the robot are in physical contact. Stretching machines, which are in constant contact with a human, require that the application of excessive force should be prevented in order to reduce the risk of foot pain. In other words, an automatic stretching machine must be able to control or restrict the force applied on the user.

Regarding Concept 2, an automatic stretching machine must respond to the individual differences between users through software, hardware, or both. This is because the conditions under which foot pain may be induced would depend on the symptoms of the individual patients. The machine should allow to tailor the stretching function for each user.

In the case of Concept 3, we refer to the standard for industrial cooperative robots, ISO/TS 15066 [26]. This standard determines the requirements for man-machine cooperative operations. For example, the cooperative operation of a robot is approved when a hand-guided device is used and the user can operate or stop the machine's operation in a single action. That is, by pushing or releasing a button. A hand-guided device (i.e., an enabling switch) can be used for stretching machines in order to prevent unintended operations.

\section{Implementation of safety measures}

Based on the risk assessments and the resulting safety concepts, the following safety measures were implemented in the stretching machine.
Risks ( $\mathrm{a}$ and $\mathrm{b}$ ) when an excessive load is applied to the foot, causing the foot to move more than natural ROM of the ankle, could result in injuries to the ankle and the muscles. Therefore, these risks must be primarily prevented. To ensure this, the pneumatic force, and the ROM of the machine's moving part, were restricted as follows. The maximum dorsiflexion angle (i.e., the ankle ROM) was restricted using a mechanical limiter for preventing excessive dorsiflexion. The maximum pneumatic force was restricted using two regulators. One of the regulators used was an air regulator (JR-08, Astroproducts, Japan), and it constrained the output of the compressor. The other was an electro-pneumatic regulator for electrically constraining the upper limit of the pneumatic force and controlling the pneumatics during stretching. The predetermined upper limit for the force applied to the foot was maintained even under the condition where either of the regulators malfunctioned.

Though foot pain can be prevented basically by the abovementioned safety measures, it would be essential that the setting of the stretching intensity is left to the user to further reduce the risk of foot pain with regard to individual differences in the ankle ROM and stiffness. This approach is debatable because it is not in line with machine safety. However, in the clinic, stretching intensity is determined based on the pain claimed by the patient, in part to prevent pain [8]. In other words, the condition is similar to that of manual stretching. In our study, we implemented the method as mentioned in "Tasks" section.

To reduce risk c of any foot pain caused by the user's involuntary operation of the machine, a three-position enabling switch commonly used in the industry was employed. When experiencing foot pain, the user must be able to immediately stop the operation of the machine by him/herself. To ensure this, the user was given the control of the machine via the above-mentioned switch. When the switch is not gripped (Position 1), the machine is in OFF state, that is, the non-operating state. When the switch is gripped (Position 2), the machine is in ON state, that is, the operating state. When the switch is gripped more strongly (Position 3 ), the machine again goes into OFF state, and this state does not change until the switch is completely released (Position 1). To maintain ON state, a continuous gripping force is necessary.

When the user releases the switch or grips it strongly, the air regulator is released to automatically reduce the pneumatic force applied to the bellows, and then the foot is moved back to a relaxed posture. Therefore, either stretching ceases or the posture is no longer one where the user experiences foot pain. This is a safety measure for risk $\mathrm{d}$. An emergency stop button was also installed for the cases where the switch does not work correctly; 
this is a safety measure for risk e. When this button is pressed, the input voltage to the electro-pneumatic regulator is cut off and the internal pressure of the bellows decreases.

For risk $f$, wherein the fingers are pinched between the gaps of the machine, the relevant parts were covered as per ISO 13854 [31]. For risk g, wherein the machine slides during operation, a rubber material was placed on the bottom of the machine as well as on the tips of its legs. Thus, friction with the floor was improved, and the slippage of the machine was reduced.

\section{Experimental verification}

\section{Objectives}

Experiments were performed on healthy young participants to verify the stretching effect of the developed stretching machine with the safety measures implemented. In general, stretching is suitable even for healthy people [32, 33].

Several studies showed reductions in the maximum voluntary contraction force of the plantar flexors (MVC) $[34,35]$ or the passive resistance [36-38] after stretching. We employed these two indices to evaluate the effectiveness of the machine. In this study, we adopted the passive resistance instead of the ankle ROM, which is often used as an index, because the results of related studies suggested that they covary $[39,40]$. Additionally, Sun et al. showed that they were correlated with each other for stroke patients [41].

\section{Participants}

Sixteen healthy young people (22.3 \pm 3.6 years old) participated in the experiments after providing written informed consent. None of the participants had injuries to their ankles or lower legs. Further, they did not perform self-stretching before the experiments. It was confirmed in advance that their calf muscles were expected to be stretched effectively by the output of the machine and the ROM of the machine moving part.

\section{Tasks}

The participants were indicated to sit on a chair with their knee fully extended for effectively stretching the gastrocnemius, which is the main muscle involved in ankle plantar flexion. Kennedy and Cresswell [42] reported that the more the knee is extended, the more the muscle is stretched when the foot is dorsiflexed. The knee angle was adjusted by changing the position and height of the chair relative to the stretching machine. Further, the machine's rotation axis was aligned with the ankle by placing a few plates below the heel.

Before starting the stretching process, the upper limit of the pneumatic force for dorsiflexion (i.e. stretching intensity) was set in the following manner. The participant operated the machine using the enabling switch. While the switch was gripped, the foot was dorsiflexed. The participant then released the switch when feeling the calf muscles stretched completely compared with his/her experience of self-stretching. The pneumatic force was set to the upper limit immediately before the switch was released. The machine's operational speed reduced as the foot was dorsiflexed, with the rate of movement dropping below $2^{\circ} / \mathrm{s}$.

For safety purposes, the machine's operation was suspended every $5 \mathrm{~min}$, and stretching was intermittently performed for a total of $25 \mathrm{~min}$. The upper limit of the pneumatic force was set every $5 \mathrm{~min}$ when the machine operation was suspended, as it was assumed that the ankle stiffness would change during stretching. Photographs of the machine in use are shown in Fig. 3.
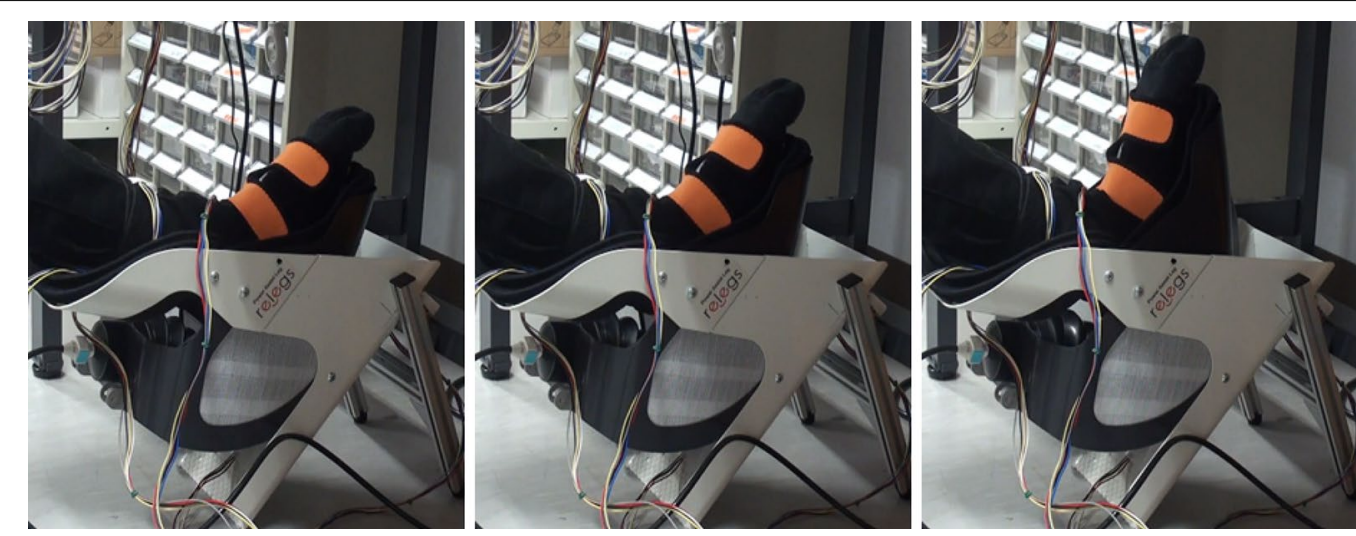

Fig. 3 Scenes of stretching. Left: In the initial stage, the foot is relaxed. Middle: In the middle stage, the foot is being dorsiflexed slowly. Right: In the final stage, the foot is maintained in the dorsiflexed posture 


\section{Measurements of maximum voluntary contraction force and passive resistance}

The MVC and the passive resistance were measured immediately before and after stretching. Each measurement took approximately $2 \mathrm{~min}$. The measurements started within 2 min after the whole stretching.

The MVC was measured using a hand-held dynamometer (MT-100, Sakai Medical Co., Ltd., Japan). This device has the functionality of a pull and push sensor, and the pull sensor function was used to measure the MVC. The belt attached to the dynamometer was fixed at the peroneal head under the knee and the thenar, and the pulling force was measured by voluntarily pulling the belt in the plantar flexion direction. During the measurements, the participant kept their foot at a dorsiflexion angle of $0^{\circ}$ with their knee fully extended. Note that the measured MVC was not the moment but the pulling force of the belt which depends on the length of the individual's lower leg and the size of their foot. The MVC was measured seven times before and after stretching. Muscle weakness due to fatigue did not affect the measurements because the mean values of first-third and fifth-seventh measurements had no significant differences ( $t$-test, pre-stretching: $t(94)=0.99, p=0.33$, post-stretching: $t(94)=0.37, p=0.71)$.

The passive resistance was measured using the push sensor function of the dynamometer and a joint angle meter (TTM-KJ, Sakai Medical Co., Ltd., Japan). The evaluator pushed at the thenar and hypothenar of the bottom of the participant's foot using the dynamometer and moved the foot up to prescribed angles. The external force required to move the relaxed foot to dorsiflexion angles of $0^{\circ}, 5^{\circ}$ and $10^{\circ}$ in the sitting position was recorded. The participants were instructed to relax their feet, and not to intentionally apply the force to the dynamometer. If the experimenter judged or the participants declared that they were not relaxed, the measurement was reperformed. The passive resistance at each angle was measured five times before and after stretching.

The standard deviations of the MVC and the passive resistance measurements were up to $15 \%$ and $14 \%$ of their means, respectively.

\section{Results}

Figure 4 shows an example of the torque generated by the machine during stretching. The torque was the maximum when the pressure reached the set upper limit. Though the internal pressure of the bellows was controlled to be constant, the inclination of the machine's moving part became larger as stretching progressed (lower graph in Fig. 4), and the torque slowly decreased as the volume
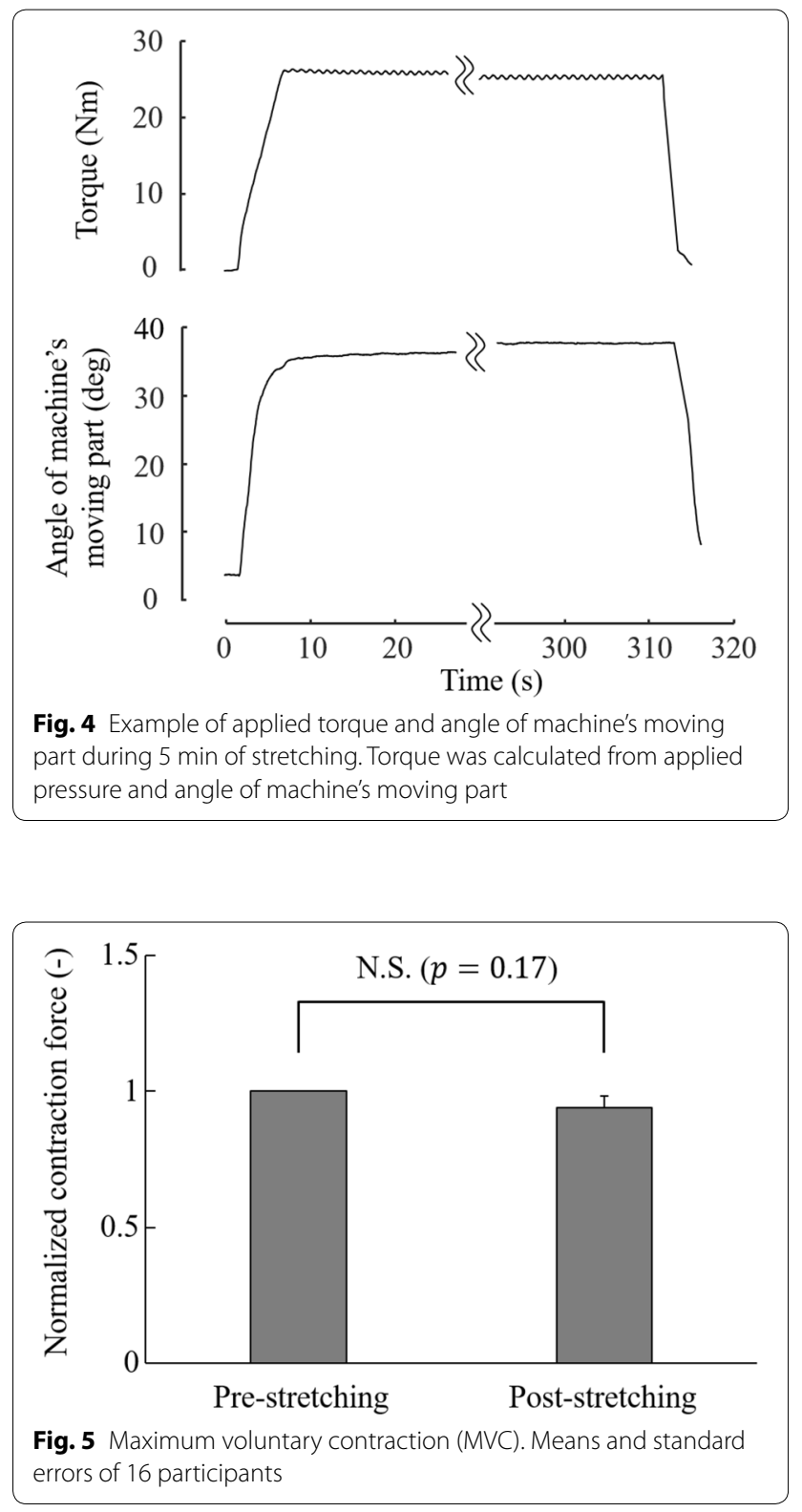

of the bellows increased. During steady state operation, the internal pressure of the bellows was kept at the value determined by each participant and was in the range of 60-140 $\mathrm{kPa}$ for all the participants. Accordingly, the maximum torque was $20-35 \mathrm{Nm}$.

Figure 5 shows the results of the MVC. The mean values normalized with respect to the MVC of each participant during pre-stretching and the standard errors are given in the figure. The MVC of 11 participants out of the total of 16 decreased after stretching by an average of $6.1 \%$. However, the mean values of all the participants of pre- and post-stretching did not exhibit any significant differences $(t(15)=1.4, p=0.17)$. 
Figure 6 shows the results of the passive resistance. The results of all 16 participants are shown except for one whose passive resistance at a dorsiflexion angle of $10^{\circ}$ could not be measured. The mean values normalized with respect to the resistance at a dorsiflexion angle of $0^{\circ}$ before stretching and the standard errors are given in the figure. After stretching, the passive resistance at $0^{\circ}, 5^{\circ}$, and $10^{\circ}$ decreased by $8.1 \%, 16.9 \%$, and $15.8 \%$, respectively. A two-way analysis of variance was performed using the pre- and post-stretching results and the measurement angles as factors. We found that the passive resistance significantly decreased after stretching $(F(1,84)=20.8$, $p<0.001)$ and had no interaction.

\section{Discussion}

Several previous studies have demonstrated that the passive resistance decreases after static stretching as the experimental results of our study confirmed. For example, Odeen and Knutsson [36] showed that the passive resistance decreased by $32 \%$ on average after stretching calf muscles of stroke patients for $30 \mathrm{~min}$. Also, it decreased by $18-19 \%[37,38]$ and $8.3 \%$ [43] after stretching the hamstring muscle for a total duration of $360 \mathrm{~s}$ and $450 \mathrm{~s}$, respectively.

On the other hand, a few studies reported that the passive resistance did not significantly decrease after stretching the hamstring muscle or the plantar flexors for the total duration of $90 \mathrm{~s}$ and $120 \mathrm{~s}$, respectively [44, 45 ], where the total duration of stretching was relatively short.

A few studies have investigated the effect of static stretching on the decrease in the muscle strength. Fowles et al. reported that the MVC decreased by $28 \%$ immediately after the stretching of the plantar flexor muscles. Furthermore, Marek et al. reported that the MVC

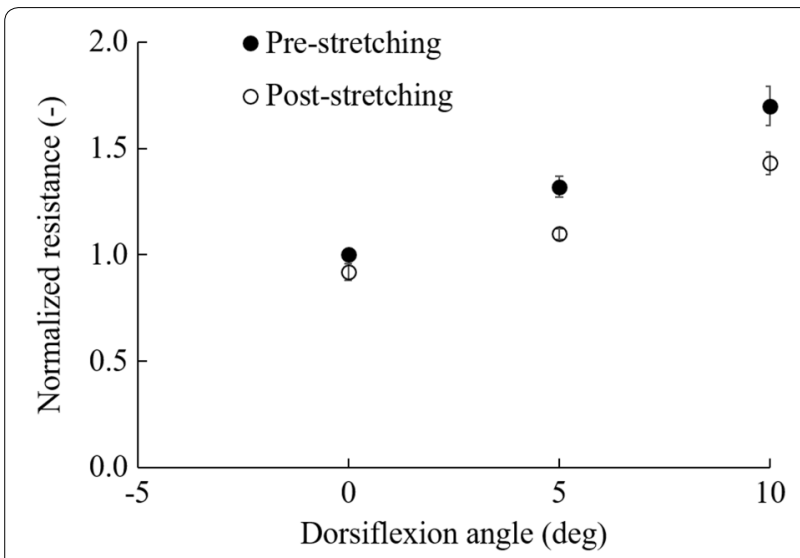

Fig. 6 Passive resistance. Passive resistance decreased significantly after stretching $(p<0.001)$. Error bars represent standard errors decreased immediately after static or proprioceptive neuromuscular facilitative stretching of the thigh muscle. The reason for the decrease in the muscle strength after static stretching has been discussed from several viewpoints [34, 35]. However, the actual mechanism remains unknown. Though it was predicted that the MVC would decrease in the present study after stretching for $5 \mathrm{~min} \times 5[46,47]$, significant differences were not observed after the stretching process. Nonetheless, it was also reported that MVC does not always decrease after static stretching of the ankle, even when the joint stiffness reduces $[48,49]$.

We proposed three safety concepts for designing automatic ankle stretching machines. Based on the results of the risk assessments of our prototype, three scenarios were judged as unacceptable risks. These were the application of an excessive stretching force on the user's foot and ankle, any unexpected operation of the machine, and individual differences in the joint deformation and stiffness. These scenarios can be considered common ones with respect to the safety requirements of stretching machines in general. Safety measures specific to machines should be implemented based on existing safety standards.

In the experiments performed, which involved young, healthy subjects, including the preliminary experiments, the prototype stretching machine was used for more than $50 \mathrm{~h}$. During this period, there were no instances of skin abrasion or foot pain during stretching. Further, there was only one incident wherein the skin was temporarily inflamed through direct contact with the belt fixing the foot. This was subsequently prevented by using a long sock. Further, while there were two instances when a part of the foot sole became slightly numb, the numbness did not remain after the experiment was over.

In the future, we have to verify the effect and safety of the machine's stretching through clinical trials involving actual patients. In addition, the safety concepts established in the present study have to be verified for ankle stretching machine other than the one we used.

\section{Conclusions}

Thus far, no commercial ankle stretching machine exists, and safety concepts of such machines have not been established. In this study, we prototyped a stretching machine for ankle dorsiflexion, and we proposed three safety concepts as the general requirements for automatic ankle stretching machines. Based on the concepts, the safety measures were implemented to the prototype machine. Further, the effectiveness was verified for healthy young participants to confirm that the machine achieved the stretching effect. The machine was used to stretch the plantar flexors, and it was observed that the 
passive resistance significantly decreased after stretching. The results indicated that the stretching machine was effective for healthy young people.

\section{Acknowledgements}

We acknowledge technical support from LAP Co., Ltd.

\section{Authors' contributions}

YS and SO are of equal contributions including experiments, analysis, and writing. NY and KI largely contributed to the risk assessment and experimental design. AY and $Y Y$ helped organize the study and paper. All authors read and approved the final manuscript.

\section{Funding}

Part of this study was supported by MEXT KAKENHI and Japan Society for the Promotion of Science (17K13108) and Aichi Medical College.

\section{Availability of data and materials}

The readers can require the corresponding author to access the experimental data by email.

\section{Competing interests}

The authors declare that they have no competing interests.

\section{Author details}

${ }^{1}$ Graduate School of Engineering, Nagoya University, Nagoya, Japan. ${ }^{2}$ Aichi Medical College, Kiyosu, Japan.

\section{Received: 12 October 2019 Accepted: 12 February 2020}

Published online: 18 February 2020

\section{References}

1. Weir D, Tingley J, Elder G (2005) Acute passive stretching alters the mechanical properties of human plantar flexors and the optimal angle for maximal voluntary contraction. Eur J Appl Physiol 93(5-6):614-623

2. Waldman G, Yang CY, Ren Y, Liu L, Guo X, Harvey RL, Roth EJ, Zhang LQ (2013) Effects of robot-guided passive stretching and active movement training of ankle and mobility impairments in stroke. NeuroRehabilitation 32(3):625-634

3. Ren Y, Wu YN, Yang CY, Xu T, Harvey RL, Zhang LQ (2017) Developing a wearable ankle rehabilitation robotic device for in-bed acute stroke rehabilitation. IEEE Trans Neural Syst Rehabil Eng 25(6):589-596

4. Zhou Z, Sun Y, Wang N, Gao F, Wei K, Wang Q (2016) Robot-assisted rehabilitation of ankle plantar flexors spasticity: a 3-month study with proprioceptive neuromuscular facilitation. Front Neurorobot 10:16

5. Toda H, Matsumoto T, Tanizaki R, Imaeda T (2016) Ankle joint pushing mechanism by stabilization of ankle position using a brace structure. J Adv Mech Des Syst Manuf 10(1):0013

6. Yamaguchi T, Toda H (2017) Proposing the safety mechanismof ankle joint pushing machine to prevent the over stretching. In: Proceedings of international conference on mechatronics, control and automation engineering, pp 133-137

7. Toda H, Matsumoto T, Sugihara S (2019) Simple geometrical analysis for mechanizing the ankle joint stretching treatment procedure of a pt using a numerical calculation. J Adv Mech Des Syst Manuf 13(2):0034

8. Yamada N, Okamoto S, Akiyama Y, Isogai K, Yamada Y (2017) Ankle stretching rehabilitation machine forequinovarus: design and evaluation from clinical aspects. In: Proceedings of IEEE international conference on systems, man, and cybernetics, pp 1687-1692

9. Kimura T, Okamoto S, Yamada N, Akiyama Y, Isogai K, Yamada Y (2017) Ankle stretching rehabilitation machine for equinovarus: automation of eversion and flexion control. In: Proceedings of IEEE international conference on systems, man, and cybernetics, pp 2696-2700

10. Saga N, Saito N (2008) Rehabilitation instrument for prevent contracture of ankle using the pneumatic balloon actuator. In: Proceedings of international conference of the IEEE engineering in medicine and biology society, pp 4294-4297
11. Cordo P, Lutsep H, Cordo L, Wright WG, Cacciatore T, Skoss R (2009) Assisted movement with enhanced sensation (AMES): coupling motor and sensory to remediate motor deficits in chronic stroke patients. Neurorehabil Neural Repair 23(1):67-77

12. Homma K, Usuba M (2011) Effects of passive motion using mechatronic system on improvement of peripheral circulation. In: Proceedings of IEEE/ SICE international symposium on system integration, pp 543-548

13. Sasanuma H, Tsukagoshi H, Okui M (2018) Socks type actuator that provides exercise for ankle and toes from the medical point of view. In: Proceedings of IEEE/ASME international conference on advanced intelligent mechatronics, pp 1228-1233

14. Yonezawa T, Nomura K, Onodera T, Ichimura S, Mizoguchi H, Takemura H (2015) Evaluation of venous return in lower limb by passive ankle exercise performed by pharad. In: Proceedings of international conference of the IEEE engineering in medicine and biology society, pp 3582-3585

15. Girone MJ, Burdea GC, Bouzit M (1999) The "Rutgers Ankle" orthopedic rehabilitation interface. In: Proceedings of the ASME haptics symposium, vol 67, pp 305-312

16. Girone MJ, Burdea GC, Bouzit M, Popescu V, Deutsch JE (2000) Othopedic rehabilitation using the "Rutgers Ankle" interface. Stud Health Technol Inform 70:89-95

17. Deutsch JE, Latonio J, Burdea GC (2001) Rehabilitation of musculoskeletal injuries using the Rutgers Ankle haptic interface: three case reports. In: Proceedings of eurohaptics conference, vol 1, pp 11-16

18. Deutsch JE, Latonio J, Burdea GC, Boian RF (2001) Post-stroke rehabilitation with the rutgers ankle system: a case study. Presence 10(4):420-435

19. Yoon J, Ryu J, Lim K-B (2006) Reconfigurable ankle rehabilitation robot for various exercises. J Robot Syst 22(S1):15-33

20. Itoh S, Kubota K, Ogata K, Tsuji T (2016) Exercise system for eccentric tibialis anterior contraction to improve ambulatory function. In: Proceedings of annual international conference of the IEEE engineering in medicine and biology society, pp 5845-5848

21. Jamwal PK, Hussain S, Ghayesh MH, Rogozina SV (2017) Adaptive impedance control of parallel ankle rehabilitation robot. J Dyn Syst Meas Control 139(11):111006-111012

22. ISO/FDIS 12100 (2010) Safety of machinery - general principles for design_risk assessment and risk reduction

23. Shiraishi Y, Okamoto S, Yamada N, Inoue K, Akiyama Y, Yamada Y (2019) Pneumatic-driven ankle stretching machine. In: Proceedings of IEEE global conference on life sciences and technologies, pp 10-11

24. Yamada N, Okamoto S, Akiyama Y, Isogai K, Kawakami K, Yamada Y (2014) Commonality and individuality in manual stretching of physical therapists for equinovarus. In: Proceedings of IEEE/SICE international symposium on system integration, pp 495-460

25. IEC 60204-1 (2016) Safety of machinery - electrical equipment of machines - part 1: general requirements

26. ISO/TS 15066 (2016) Robots and robotic devices-collaborative robots

27. ISO 13482 (2014) Robots and robotic devices-safety requirements for personal care robots

28. Mao X, Yamada Y, Akiyama Y, Okamoto S (2017) Characteristics of dummy skin contact mechanics during developing process of skin abrasion trauma. Tribol Lett 65(4):133

29. Mao X, Yamada Y, Akiyama Y, Okamoto S, Yoshida K (2017) Safety verification method for preventing friction blisters during utilization of physical assistant robots. Adv Robot 31(13):680-694

30. Harvey LA, Katalinic OM, Herbert RD, Moseley AM, Lannin NA, Schurr K (2017) Stretch for the treatment and prevention of contractures. Cochrane Database Syst Rev, 1

31. ISO 13854 (2017) Safety of machinery — minimum gaps to avoid crushing of parts of the human body

32. Marshall PW, Cashman A, Cheema BS (2011) A randomized controlled trial for the effect of passive stretching on measures of hamstring extensibility, passive stiffness, strength, and stretch tolerance. J Sci Med Sport 14(6):535-540

33. McNair P, Dombroski E, Hewson D, Stanley S (2001) Stretching at the ankle joint: viscoelastic responses to holds and continuous passive motion. Med Sci Sports Exerc 33(3):354-358

34. Fowles JR, Sale DG, Macdougall JD (2000) Reduced strength after passive stretch of the human plantarflexors. J Appl Physiol 89(3):1179-1188

35. Marek SM, Cramer JT, Fincher AL, Massey LL, Dangelmaier SM, Purkayastha S, Fitz KA, Culbertson JY (2005) Acute effects of static and 
proprioceptive neuromuscular facilitation stretching on muscle strength and power output. J Athletic Train 40(2):94-103

36. Odeen I, Knutsson E (1981) Evaluation of the effects of muscle stretch and weight load in patients with spastic paraplegia. Scand J Rehabil Med 13(4):117-121

37. Magnusson SP, Simonsen EB, Aagaard P, Gleim GW, McHugh MP, Kjaer M (1995) Viscoelastic response to repeated static stretching in the human hamstring muscle. Scand J Med Sci Sports 5(6):342-347

38. Magnusson SP, Simonsen EB, Aagaard P, Kjaer M (1996) Biomechanical responses to repeated stretches in human hamstring muscle in vivo. Am J Sports Med 24(5):622-628

39. Mizuno T, Matsumoto M, Umemura Y (2013) Viscoelasticity of the muscletendon unit is returned more rapidly than range of motion after stretching. Scand J Med Sci Sports 23(1):23-30

40. Morse Cl, Seynnes OR, Maganaris CN, Jones DA (2008) The acute effect of stretching on the passive stiffness of the human gastrocnemius muscle tendon unit. J Physiol 586(1):97-106

41. Chung SG, Van Rey E, Bai Z, Zhang LQ (2004) Biomechanic changes in passive properties of hemiplegic ankles with spastic hypertonia. Arch Phys Med Rehabil 85(10):1638-1646

42. Kennedy PM, Cresswell AG (2001) The effect of muscle length on motorunit recruitment during isometric plantar flexion in humans. Exp Brain Res 137(1):58-64
43. McHugh MP, Nesse M (2008) Effect of stretching on strength loss and pain after eccentric exercise. Med Sci Sports Exerc 40(3):566-573

44. Magnusson SP, Aagaard P, Nielson JJ (2000) Passive energy return after repeated stretches of the hamstring muscle-tendon unit. Med Sci Sports Exerc 32(6):1160-1164

45. Muir IW, Chesworth BM, Vandervoort AA (1999) Effect of a static calfstretching exercise on the resistive torque during passive ankle dorsiflexion in healthy subjects. J Orthop Sports Phys Ther 29(2):106-113

46. Kay AD, Blazevich AJ (2012) Effect of acute static stretch on maximal muscle performance: a systematic review. Med Sci Sports Exerc 44(1):154-164

47. Simic L, Sarabon N, Markovic G (2013) Does pre-exercise static stretching inhibit maximal muscular performance? A meta-analytical review. Scand J Med Sci Sports 23(2):131-148

48. Kubo K, Kanehisa H, Kawakami Y, Fukunaga T (2001) Influence of static stretching on viscoelastic properties of human tendon structures in vivo. J Appl Physiol 90(2):520-527

49. Papadopoulos C, Kalapotharakos VI, Noussios G, Meliggas K, Gantiraga E (2006) The effect of static stretching on maximal voluntary contraction and force-time curve characteristics. J Sport Rehabil 15(3):185-194

\section{Submit your manuscript to a SpringerOpen ${ }^{\odot}$ journal and benefit from:}

- Convenient online submission

- Rigorous peer review

- Open access: articles freely available online

- High visibility within the field

- Retaining the copyright to your article

Submit your next manuscript at $\boldsymbol{\nabla}$ springeropen.com 